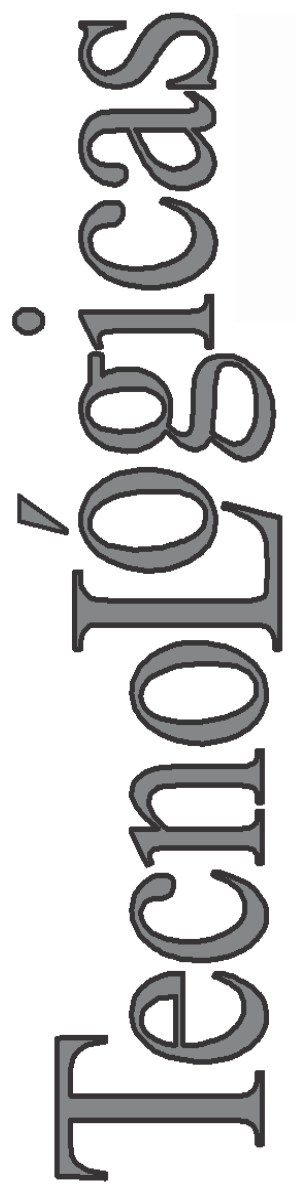

\title{
Evaluación de la Adhesión de una Suspensión MacPherson bajo la Norma EuSAMA en un Modelo Matemático y uno Multicuerpo
}

\author{
Adherence Evaluation of a \\ MacPherson Suspension under \\ EuSAMA Norm in a Mathematical \\ Model and one Multibody
}

Juan J. Arbeláez-Toro ${ }^{1}$

Carlos M. Rodríguez-Ledesma ${ }^{2}$ Diego A. Hincapié-Zuluaga ${ }^{3}$

Edwar A. Torres-Lopez ${ }^{4}$

1 Grupo de Investigación en Materiales Avanzados y Energía, Instituto Tecnológico Metropolitano, Medellín-Colombia juanarbelaez@itm.edu.co

2 Grupo de Investigación en Materiales Avanzados y

Energía, Instituto Tecnológico Metropolitano, Medellín-Colombia carlosrodriguez@itm.edu.co

3 Grupo de Investigación en Materiales Avanzados y

Energía, Instituto Tecnológico Metropolitano, Medellín-Colombia diegohincapie@itm.edu.co

4 Grupo de Investigación en Materiales Avanzados y

Energía, Instituto Tecnológico Metropolitano, Medellín-Colombia edwartorres@itm.edu.co 


\title{
Arbeláez et al. / Evaluación de la Adhesión de una Suspensión MacPherson bajo la Norma EuSAMA en un Modelo Matemático y uno Multicuerpo
}

\section{Resumen}

En este trabajo se realiza una simulación computacional, para dar respuesta a un problema de dinámica asociado a la evaluación de la adhesión en sistemas de suspensión. El proceso inicia con el levantamiento de las geometrías más representativas de un sistema MacPherson de un Nissan Sentra B13, donde cada uno de los dispositivos se crea y ensambla en un software CAD para dar solución dinámica en un paquete CAE multicuerpo. Posteriormente se crea un modelo matemático cuyas ecuaciones diferenciales se generan fundamentadas en la segunda ley de Newton y se resuelven en Simulink de Matlab®. Finalizado el proceso de elaboración de los modelos se alimentan las variables con la información precisa del vehículo de estudio para obtener las gráficas que dan respuesta al protocolo de la prueba EuSAMA (European Shock Absorber Manufaturers Association) para el análisis de la adhesión. Los resultados obtenidos evidencian que los modelos desarrollados son confiables cuando se comparan con la prueba experimental; además, se observa que la disminución del coeficiente de amortiguamiento compromete la adhesión del vehículo en la vía, afectando la estabilidad y maniobrabilidad.

\section{Palabras clave}

Adhesión; modelo matemático; modelo multicuerpo; suspensión MacPherson; EuSAMA.

\begin{abstract}
A computational simulation is Implemented, in order to response to a problem of dynamics associated With The assessment of adherence in suspension systems. The process begins with the lifting of the most representative geometries of a MacPherson system of a Nissan Sentra B13, where each of the devices is created and assembled into a CAD software to give a dynamic solution on a CAE multibody package. Afterwards a mathematical model was created whose differential equations are generated substantiated on Newton's second law and this are resolved using MatlabSimulink applications. Once the model developing process is over, the variables are fed with accurate information of the studied vehicle to obtain the graphs that give an answer to EuSAMA (European Shock Absorber Manufacturers Association) test protocol for the adherence analysis. The results presented show the reliability of the developed models when compared with the experimental test; furthermore, it demonstrates that the decrease of the damping coefficient compromises the vehicle's adherence on the track, affecting its stability and maneuverability.
\end{abstract}

\section{Keywords}

Adherence; mathematical model; multibody model; MacPherson suspension; EuSAMA. 


\section{INTRODUCCIÓN}

La European Shock Absorber Manufaturer, EuSAMA (1976), define la adhesión como la "Relación entre el mínimo valor de la fuerza vertical ejercida por una rueda, estimulada con una frecuencia de 0 a $25 \mathrm{~Hz}$, y la misma fuerza medida a $0 \mathrm{~Hz}$, (Fig. 1).

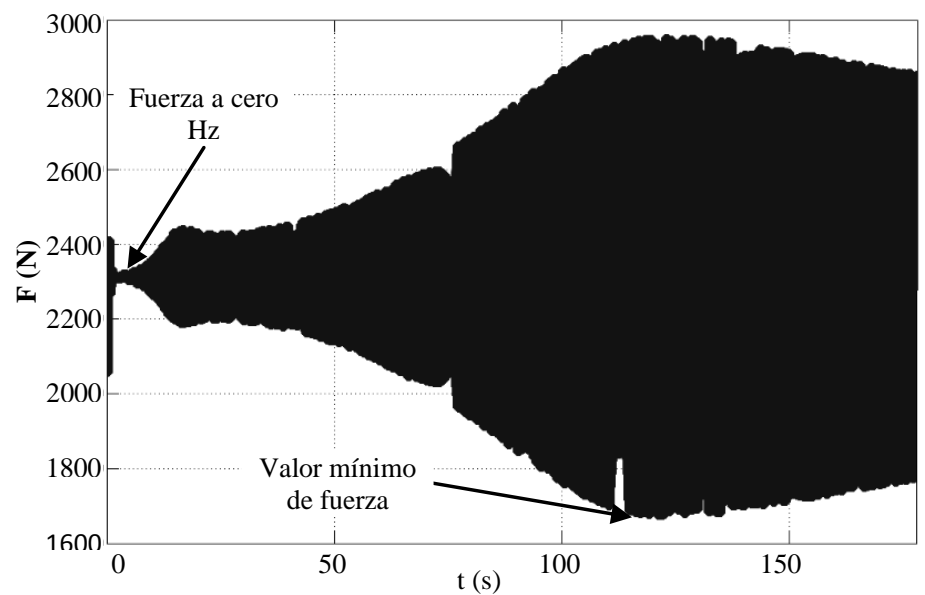

Fig. 1. Diagrama de fuerza de contacto vs frecuencia. Fuente: Autores

Este valor indica el estado actual de la suspensión, al considerar los siguientes rangos (Tabla 1).

Tabla 1. Interpretación de la EuSAMA. Fuente: Autores

\begin{tabular}{cl}
\hline Medida de Adhesión & Interpretación de la EuSAMA \\
\hline $61 \%$ a $100 \%$ & Excelente contacto dinámico de la llanta \\
$41 \%$ a $60 \%$ & Buen contacto dinámico de la llanta \\
$21 \%$ a $40 \%$ & Aceptable contacto dinámico de la llanta \\
$1 \%$ a $20 \%$ & Pobre contacto dinámico de la llanta \\
$0 \%$ & Ningún contacto dinámico de la llanta \\
\hline
\end{tabular}

Actualmente existen diferentes fabricantes de equipos comerciales para ensayos de suspensiones bajo la norma EuSAMA; ROBORTEM ${ }^{\circledR}$ afirma que, para medir el valor de la adhesión en un vehiculo, el proceso se lleva acabo, por medio de la ubicación de 
cada una de las ruedas en la plataforma vibratoria con el fin de analizar el contacto establecido entre ambos elementos. Se considera que la suspensión del vehículo está en buenas condiciones si todas las ruedas superan el $45 \%$ de adhesión y la diferencia entre dos del mismo eje es menor al 15\%. Calvo et al. (2005) han determinado coeficientes de amortiguamiento para sistemas de suspensión a partir de las gráficas resultantes de la prueba EuSAMA, como criterio para evaluar vehículos que no han pasado esta prueba, aun gozando de sistemas óptimos de amortiguación.

Actualmente se utilizan modelos computacionales y matemáticos para dar solución a problemas asociados al desempeño y diseño de sistemas de suspensión. Liu et al., (2006) optimizaron el diseño de una suspensión MacPherson con muelles de carga lateral. Fallah et al., (2008) realizaron el análisis cinemático y dinámico de una suspensión MacPherson usando el método de la matriz de desplazamiento; mientras Colombo et al., (2009) analizaron las fallas inusuales en suspensiones MacPherson; Zhu \& Niu (2010) generaron una co-simulacion de una suspensión que caracterizaron por medio de banco de ensayos de amortiguadores; Kustnetsov et al. (2011) evaluaron las mejoras de una suspensión al introducir un dispositivo inerte utilizando un modelo de cuarto de vehículo, mientras que Karen et al. (2011) fabricaron una herramienta para evaluar las características de confort sobre la marcha del vehículo.

En este trabajo se reproducen las condiciones del ensayo EuSAMA para una suspensión del tipo MacPherson de un Nissan Sentra B13, en un modelo virtual multicuerpo y un modelo matemático, para evaluar los valores de adhesión para diferentes coeficientes de amortiguamiento. Finalmente, los resultados obtenidos se compararon con las pruebas de un ensayo real del vehículo en estudio.

\section{METODOLOGía}

El diseño del modelo multicuerpo se inicia con la toma de las medidas más representativas de una suspensión MacPherson de un Nissan Sentra B13, como se presenta en la Fig. 2. 


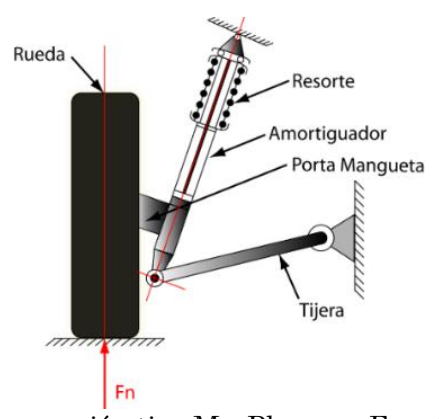

Fig. 2. Esquema de la suspensión tipo MacPherson. Fuente: Arbeláez et al., 2007

Para ser elaborados y ensamblados los elementos, fue utilizado el entorno CAD (Computer Aided Design) del software Siemens $\mathrm{Nx}^{\circledR}$, junto a otros sólidos que representa la masa suspendida de un cuarto de vehículo y el mecanismo excéntrico que genera las oscilaciones como dicta la norma EuSAMA (Fig. 3).

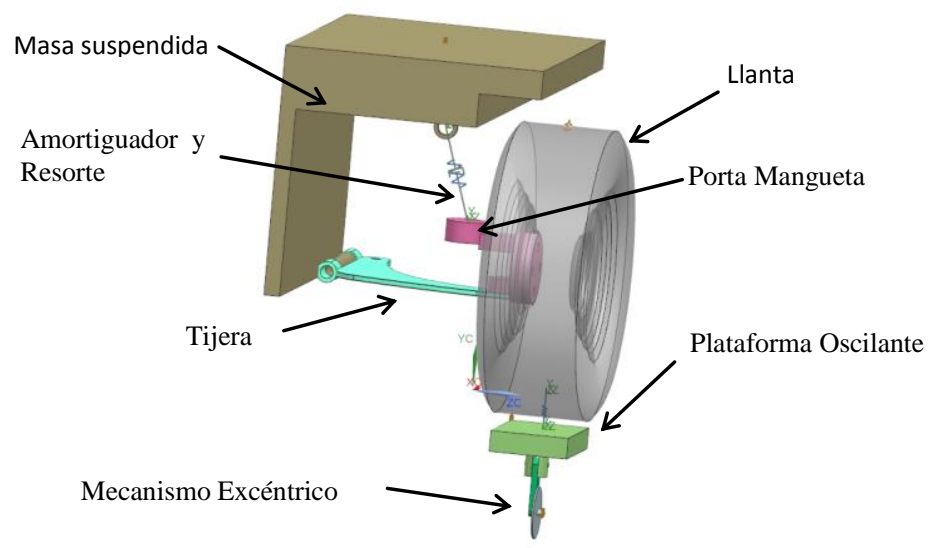

Fig. 3. Modelo CAD suspensión MacPherson. Fuente: Autores

El modelo CAD se lleva al entorno Motion del software Siemens $\mathrm{Nx}{ }^{\circledR}$, donde se definen los eslabones y las juntas cinemáticas, según ISO 3952-1 (2004). En la Fig. 4a, se observan los siguientes pares cinemáticos: 1 y 3 , par clase v (deslizador); éstos solo permiten el movimiento vertical de la masa suspendida y la plataforma. 2 par iv clase (paralelo); admite el movimiento lineal 
de la llanta paralelo a la masa suspendida. 4 Par clase iii (esférica o rótula); permite rotación en cualquier eje entre la tijera y el porta mangueta. 5, 6, 7, y 9 v clase (cilíndrica); concede rotación en un solo eje entre la masa suspendida y la tijera, entre la biela y la plataforma, y el motor y leva respectivamente. 10, es unión rígida entre la llanta y el porta mangueta. 11, par clase iii (plana); permite el movimiento de la llanta en el plano definido por su eje. Además, en la Fig. 4a, marcado con el número 12, se observa el amortiguador y resorte del sistema de suspensión. La entrada de movimiento al sistema plataforma suspensión se realiza por la junta 7 en un barrido de frecuencia de 0 a $25 \mathrm{~Hz}$.

En la elaboración del modelo matemático se hace una representación de un cuarto de vehículo, según modelos pasivos de resorte y amortiguador (Popp \& Schiehlen, 2010), como se observa en la Fig. 4b.

a)

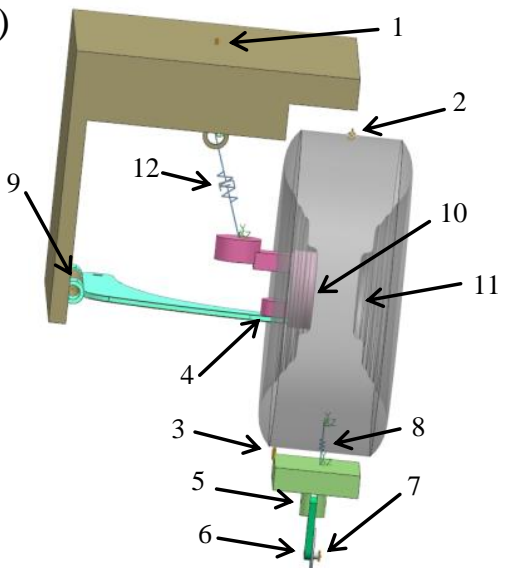

b)

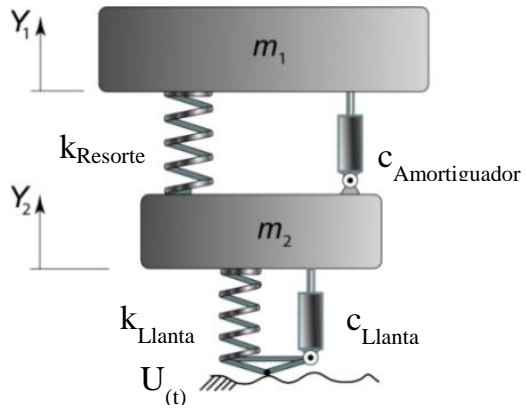

Fig. 4. a) Modelo Multicuerpo suspensión Nissan Sentra B13. Fuente: Autores. b) Modelo suspensión MacPherson. Fuente: Arbeláez et al., 2007

El modelo se representa de la siguiente manera: masa $m_{1}$ que es la masa suspendida de un cuarto de vehículo; masa $m_{2}$ que es la masa no suspendida (llanta, amortiguador, resorte y tijera); constante $k_{1}$, del resorte; constante $c_{1}$, del amortiguador; constante $k_{2}$, del resorte de la llanta; constante $c_{2}$, coeficiente de amortiguación de la llanta. 
Las ecuaciones diferenciales (1) y (2) se obtienen aplicando la segunda ley de Newton, en cada una de las masas. Con el conjunto de $m_{1}, k_{1}$ y $c_{1}$ y del conjunto de $m_{2}, k_{1}, C_{1}, k_{2}$ y $c_{2}$.

$$
\begin{aligned}
& m_{1} \ddot{y}_{1}=-k_{1}\left(y_{1}-y_{2}\right)-c_{1}\left(\dot{y}_{1}-\dot{y}_{2}\right) \\
& m_{2} \ddot{y}_{2}=-k_{1}\left(y_{2}-y_{1}\right)-c_{1}\left(\dot{y}_{2}-\dot{y}_{1}\right)-k_{2}\left(y_{2}-u\right)-c_{2}\left(\dot{y}_{2}-u\right)
\end{aligned}
$$

Después de obtener las ecuaciones diferenciales que describen el comportamiento dinámico de las suspensión en un cuarto de vehículo, la solución se realiza en Simulink de Matlab®, donde el número de integradores a usar es el orden de la ecuación diferencial, colocando la derivada de mayor orden igual a la suma de los términos que contienen derivadas de orden inferior afectadas por los coeficientes (Fig. 5).

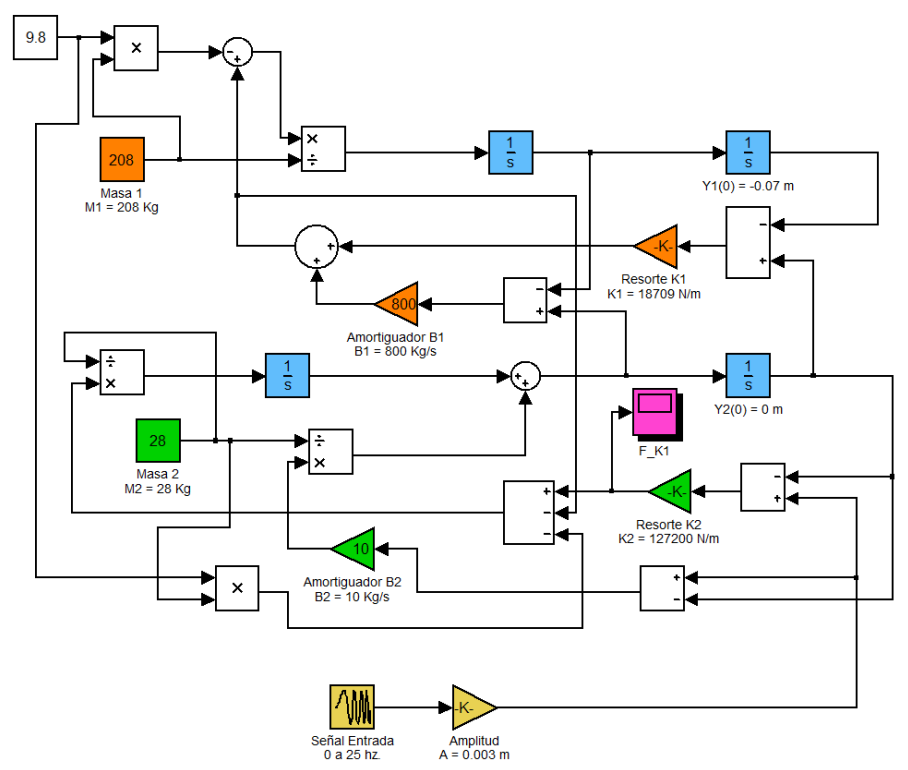

Fig. 5. Modelo dinámico cuarto vehículo. Fuente: Autores 
Los parámetros que alimentan el modelo matemático y multicuerpo de un cuarto de vehículo de un Nissan Sentra B13 se muestran en la Tabla 2; es importante destacar que ambos modelos consideran la precarga del resorte al interior del sistema de suspensión.

Tabla 2. Parámetros cuarto de vehículo. Fuente: Autores

\begin{tabular}{|c|c|c|c|}
\hline Parámetro & Valor & Unidad & Descripción \\
\hline $\begin{array}{l}\mathrm{m}_{1}=\text { masa suspendi- } \\
\mathrm{da}\end{array}$ & 208 & $\mathrm{~kg}$ & $\begin{array}{l}\text { Equivalente a la masa de un } \\
\text { cuarto de vehículo (Nissan Sentra } \\
\text { B13) }\end{array}$ \\
\hline $\begin{array}{l}\mathrm{m}_{2}=\text { masa no sus- } \\
\text { pendida }\end{array}$ & 28 & $\mathrm{~kg}$ & $\begin{array}{l}\text { Equivalente a la masa de la } \\
\text { llanta, tijera y portamangeta } \\
\text { suspensión (Nissan Sentra B13) }\end{array}$ \\
\hline $\begin{array}{l}\mathrm{k}_{1}=\text { constante del } \\
\text { resorte }\end{array}$ & 18709 & $\mathrm{kN} / \mathrm{m}$ & $\begin{array}{l}\text { Según Carvill (2003) ecuación } \\
\text { para determinar la constante de } \\
\text { una espiral }\end{array}$ \\
\hline $\begin{array}{l}\mathrm{k}_{2}=\text { constante de la } \\
\text { llanta }\end{array}$ & 127200 & $\mathrm{kN} / \mathrm{m}$ & $\begin{array}{l}\text { Obtenido por medio de ensayos a } \\
\text { tensión y compresión de una } \\
\text { llanta 185/65 R13 según Reimpell } \\
\text { (2003) }\end{array}$ \\
\hline $\begin{array}{l}\mathrm{c}_{1}=\text { coeficiente } \\
\text { amortiguación de la } \\
\text { llanta }\end{array}$ & 100 & $\mathrm{~kg} / \mathrm{s}$ & Según Calvo et al. (2005) \\
\hline
\end{tabular}

\section{RESULTADOS Y DISCUSIÓN}

En el modelo multicuerpo y en el modelo matemático se cargan las variables definidas en la Tabla 2 , se programa una entrada de barrido de frecuencias de 0 a $25 \mathrm{~Hz}$ para una oscilación de $\pm 3 \mathrm{~mm}$, como recomienda la EuSAMA y se realiza el análisis de la adhesión para tres coeficientes lineales de amortiguación $(800 \mathrm{~kg} / \mathrm{s}$, $1400 \mathrm{~kg} / \mathrm{s}$ y $2000 \mathrm{~kg} / \mathrm{s}$ ); cada uno de los modelos se grafican como se muestra en la Fig. 6 y la Fig. 7. En la Fig. 6 y la Fig. 7, se observan dos frecuencias de resonancia; la primera, corresponde a la masa suspendida y la segunda a la masa no suspendida. De las gráficas se toman el mínimo valor de fuerza (fuerza dinámica), la fuerza estática y el valor de frecuencia para cada uno de los coeficientes de amortiguación lineal con el fin de analizar la adhesión en el sistema de suspensión. 


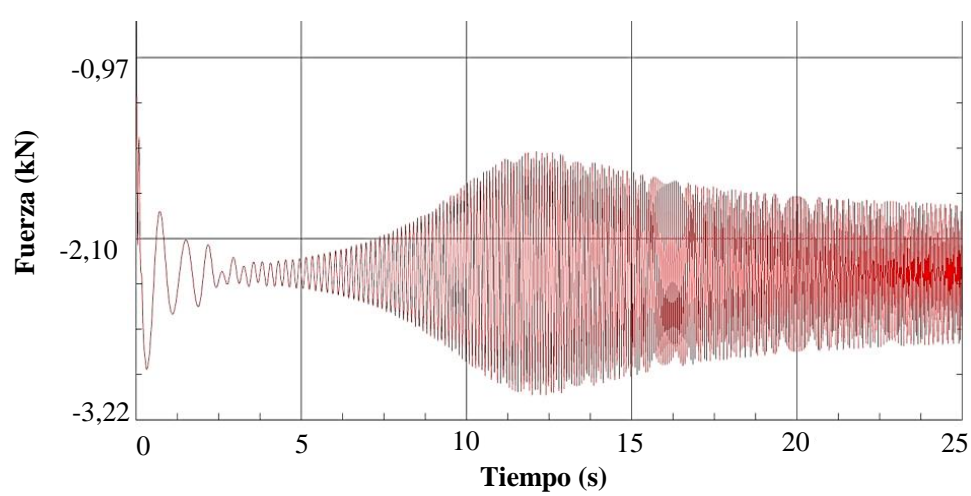

Fig. 6. Resultado ensayo EuSAMA modelo multicuerpo. Fuente: Autores

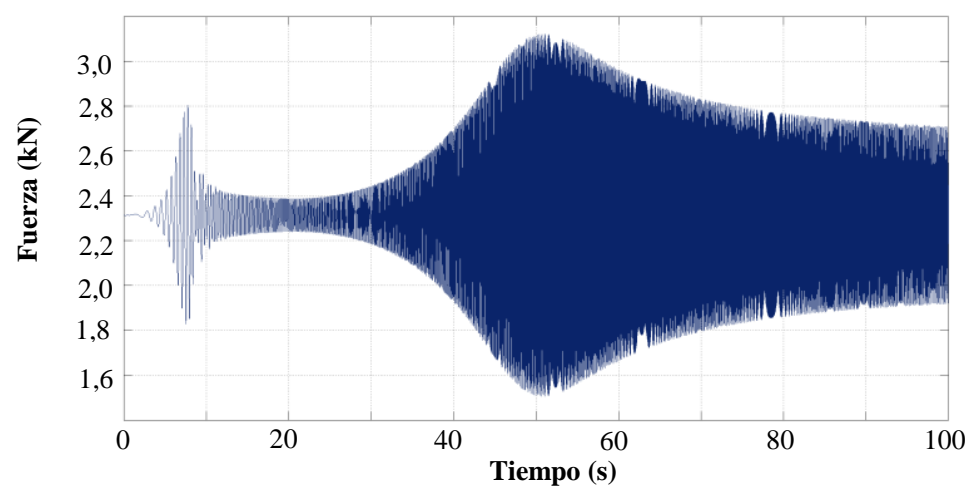

Fig. 7. Resultado ensayo EuSAMA modelo matemático. Fuente: Autores

Los datos de todas las pruebas realizadas se condensan en la Tabla 3, para el modelo multicuerpo, y, en la Tabla 4, para el modelo matemático.

Tabla 3. Respuesta modelo multicuerpo norma EuSAMA. Fuente: Autores

\begin{tabular}{ccccc}
\hline $\begin{array}{c}\text { Coeficiente de } \\
\text { amortiguación }\end{array}$ & $\begin{array}{c}\text { Fuerza } \\
\text { Dinámica } \\
\text { FD (N) }\end{array}$ & $\begin{array}{c}\text { Fuerza } \\
\text { Estática } \\
\text { FE (N) }\end{array}$ & $\begin{array}{c}\text { Frecuencia } \\
(\mathrm{Hz})\end{array}$ & $\begin{array}{c}\text { Valor de la } \\
\text { adhesión } \\
\text { EuSAMA } \\
(\text { FD/FE *100\%) }\end{array}$ \\
\hline $800 \mathrm{~kg} / \mathrm{s}$ & 1555 & 2314 & 12,1 & $67,19 \%$ \\
$1400 \mathrm{~kg} / \mathrm{s}$ & 1736 & 2314 & 11,9 & $75,02 \%$ \\
$2000 \mathrm{~kg} / \mathrm{s}$ & 1804 & 2314 & 11.9 & $77,96 \%$ \\
\hline
\end{tabular}


Arbeláez et al. / Evaluación de la Adhesión de una Suspensión MacPherson bajo la Norma EuSAMA en un Modelo Matemático y uno Multicuerpo

Tabla 4. Respuesta modelo matemático norma EuSAMA. Fuente: Autores

\begin{tabular}{ccccc}
\hline $\begin{array}{c}\text { Coeficiente de } \\
\text { amortiguación }\end{array}$ & $\begin{array}{c}\text { Fuerza } \\
\text { Dinámica } \\
\text { FD (N) }\end{array}$ & $\begin{array}{c}\text { Fuerza } \\
\text { Estática } \\
\text { FE (N) }\end{array}$ & $\begin{array}{c}\text { Frecuencia } \\
(\mathrm{Hz})\end{array}$ & $\begin{array}{c}\text { Valor de la } \\
\text { adhesión } \\
\text { EuSAMA } \\
(\text { FD/FE *100\%) }\end{array}$ \\
\hline $800 \mathrm{~kg} / \mathrm{s}$ & 1398 & 2314 & 12,5 & $60,41 \%$ \\
$1400 \mathrm{~kg} / \mathrm{s}$ & 1683 & 2314 & 12,4 & $72,73 \%$ \\
$2000 \mathrm{~kg} / \mathrm{s}$ & 1780 & 2314 & 12,4 & $76,92 \%$ \\
\hline
\end{tabular}

Obtenidos los valores de adhesión, se determina el estado del sistema de suspensión según la Tabla 1 que para este caso corresponde a: Excelente contacto dinámico para los valores de $800 \mathrm{~kg} / \mathrm{s}$, $1400 \mathrm{~kg} / \mathrm{s}$ y $2000 \mathrm{~kg} / \mathrm{s}$. De la Tabla 1; el valor de la frecuencia a la que sucede la mínima fuerza en los dos sistemas es aproximadamente el mismo $(12 \mathrm{~Hz})$, pero la magnitud de esta fuerza es diferente, siendo mayor en el modelo matemático, diferencia que se justifica porque este modelo no incluye los aspectos geométricos del sistema. En relación con los resultados de adhesión; ambos modelos son afines a valores mayores de coeficiente de amortiguamiento, pero discrepan en un pequeño porcentaje a valores menores. En la Fig. 8 se aprecia una gráfica real de una suspensión de un Nissan Sentra realizado en el banco de pruebas de la Universidad Tecnológica de Pereira; los resultados de este ensayo se ajustan a los obtenidos por medio del modelo matemático y el modelo multicuerpo utilizando un coeficiente de amortiguación de $1400 \mathrm{~kg} / \mathrm{s}$, propio de este tipo de suspensión

\section{CONCLUSIONES}

Se elaboró un modelo multicuerpo y un modelo matemático de un cuarto de vehículo y una plataforma oscilante, para la evaluación de la adhesión bajo la norma EuSAMA. Se observó que las respuestas de los modelos matemáticos y multicuerpo son similares en fuerza estática, fuerza dinámica y frecuencia para los diferentes coeficientes de amortiguamiento.De los datos obtenidos se encuentra que a medida que disminuye el coeficiente de fricción se pierde adhesión en el sistema de suspensión, afectando la adherencia del vehículo a la vía y la maniobrabilidad. 


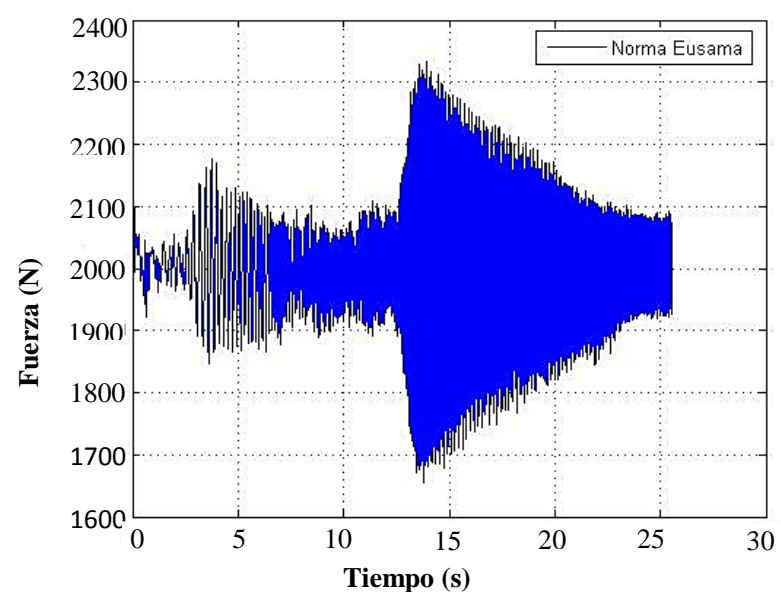

Fig. 8. Curva real de una suspensión de Nissan Sentra determinado en banco de pruebas. Fuente: Autores

Ambos modelos son afines en la evaluación de la adhesión, a valores mayores de coeficiente de amortiguamiento, pero discrepan en un pequeño porcentaje a valores menores. Los valores de adhesión, para el coeficiente de amortiguación de $1400 \mathrm{~kg} / \mathrm{s}$ de los modelos desarrollados en este trabajo, se aproximan a la respuesta del modelo experimental, validando la veracidad del modelo matemático y el modelo computacional. La norma EuSAMA valida el estado general del conjunto de suspension, pero no tiene la capacidad de determinar daños en componentes específicos; para el caso de suspensiones MacPherson, valores inferiores de adhesión se asocian a daños en el amortiguador.

\section{REFERENCIAS}

Arbelález, J.J., Quinter, J.P. \& Calle, G. Modelado, diseño y construcción de un banco de pruebas para el análisis de la adhesión en la evaluación en suspensiones de vehículos livianos bajo la norma European Shock absorber Manufacturers Association (EuSAMA), $8^{\circ}$ Congreso Iberoamericano de Ingeniería Mecánica (CIBIM 2007), Cusco, Perú.

Calvo, J. A., Diaz, V. \& San Roman, J. L. (2005). Establishing inspection criteria to verify the dynamic. Int. J. Vehicle Design, 38(4), 290-306. 
Carvill, J. (2003). Mechanical Engineer`s data handbook, 32-35, Butterworth Heinemann, Oxford, Great Britain.

Colombo, D., Gobbi, M. Mastinu, G. \& Pennate, M. (2009). Analysis of an unusual MacPherson suspension failure. Engineering failure analysis, 16, 1000-1010.

EuSAMA, (1976). Recommendation for performance test specification of car vehicle suspension testing system. EUSAMA-TS-02-76.

Fallah, M., Mahzoon, M. \& Eghtesad, M. (2008). Kinematical and dynamical analysis of MacPherson suspension using displacement matrix method. Iranian Journal of Science \& Technology, Transaction B, Engineering, 32(B4), 325-339.

Karen, İ., Kaya, N., Öztürk F. Korkmaz İ., Yıldızhan, M. \& Yurttaş, A. (2012). A design tool to evaluate the vehicle ride comfort characteristics: modeling, physical testing, and analysis. Int j adv manuf technol, $60,755-763$.

Kuznetsov, A., Mammadov, M., Sultan, I., \& Hajilarov, E. (2011). Optimization of improved suspension system with inerter device of the quarter-car model in vibration analysis. Arch appl mech, 81, 1427-1437.

Liu, J., Zhuang, D. J., Yu, F. \& Lou L. M. (2008). Optimized design for a MacPherson strut suspension with side load springs. International Journal of Automotive Technology, 9(1), 29-35.

Pacejka, H. (2006). Tyre and Vehicles Dynamics. Netherland: TNO. 621.

Popp, K. \& Schiehlen, W. (2010). Ground vehicle dynamics. 99.

Reimpell, J. (2001). The Automotive Chassis: Engineering Principles, 2a Edition, 62-66, Butterworth Heinemann, England.

UNE-EN ISO 3952-1/A1. (2004). Kinematic diagrams - graphical symbols - part 1 (ISO 3952-1:1981/Amd 1:2002). 8.

Zhu, R., \& Niu, Limin. (2010). Research on co-simulation and test of semiactive suspension. Second International Conference on Computer Modeling and Simulation. 